\title{
Multiple Cranial Nerve Palsies in a Pediatric Case of Lemierre's Syndrome due to Streptococcus viridans
}

\author{
Samantha Novotny $\mathbb{D D}^{1},{ }^{1}$ Kenneth Serrano, ${ }^{1}$ Danielle Bazer, ${ }^{2}$ and Louis Manganas ${ }^{2}$ \\ ${ }^{1}$ Renaissance School of Medicine at Stony Brook University, 101 Nicolls Road, Stony Brook, New York 11794, USA \\ ${ }^{2}$ Department of Neurology at Stony Brook University Hospital, 101 Nicolls Road Stony Brook, New York 11794, USA
}

Correspondence should be addressed to Samantha Novotny; samantha.novotny@stonybrookmedicine.edu

Received 15 July 2021; Accepted 15 October 2021; Published 26 October 2021

Academic Editor: Dominic B. Fee

Copyright ( $) 2021$ Samantha Novotny et al. This is an open access article distributed under the Creative Commons Attribution License, which permits unrestricted use, distribution, and reproduction in any medium, provided the original work is properly cited.

\begin{abstract}
Background. Lemierre's syndrome is a rare condition of internal jugular vein thrombosis following oropharyngeal infection. While it usually results from Fusobacterium necrophorum infection, atypical cases associated with other pathogens have been reported. Objective. To describe a unique case of pediatric Lemierre's syndrome with Streptococcus viridans infection resulting in cavernous sinus thrombosis and oculomotor, trochlear, and abducens nerve palsies. Case Report. A 14-year-old female initially presented after six days of fever, myalgias, and sore throat and was admitted for hyperbilirubinemia and acute kidney injury. She developed a fixed, dilated pupil with complete ophthalmoplegia, ptosis, and severe pain. Imaging revealed retromandibular space abscess, external and internal jugular vein thrombosis, cavernous sinus thrombosis, internal carotid artery stenosis, pulmonary embolism, and bilateral pneumonia. She was diagnosed with Lemierre's syndrome with cultures positive for Streptococcus viridans and treated with a combination of antibiotics and anticoagulation. Conclusion and Relevance. Both antibiotics and anticoagulation were effective management for this Lemierre's syndrome patient with cavernous sinus thrombosis. Early diagnosis and treatment of Lemierre's syndrome is essential. A multidisciplinary treatment team is beneficial for managing the sequelae of this condition.
\end{abstract}

\section{Introduction}

Lemierre's syndrome is a rare sequela of oropharyngeal infection in previously healthy young adults resulting in thrombosis of the internal jugular vein and septic embolism [1]. The most common offending organism is Fusobacterium necrophorum [2], although other organisms have been reported including Staphylococcus aureus and several strains of Streptococcus [3-9]. We describe a unique case of pediatric Lemierre's syndrome caused by Streptococcus viridans with multiorgan involvement and progression to cavernous sinus thrombosis with cranial nerves III, IV, and VI palsies. While Lemierre's syndrome associated with Streptococcus viridans infection has previously been described, our patient with development of complete ophthalmoplegia demonstrates an underreported sequela of this condition.

\section{Case Presentation}

A 14-year-old female with no medical history presented to the emergency department with a six-day history of fever, myalgias, pharyngitis, throbbing headache, and left eye ptosis. Her symptoms were associated with diplopia, blurred vision, photophobia, and phonophobia. Her neurological exam was significant for visual acuity of 20/200 in the left eye with an afferent pupillary defect. There were also cranial nerve III, IV, and VI palsies.

Throat swab cultures grew Candida dubliniensis and viridans group alpha hemolytic Streptococcus. Her hospital course was complicated by left retromandibular space abscess, left external jugular vein thrombosis, partial left internal jugular vein thrombosis, bilateral lower lobe pneumonia, and multiple pulmonary consolidations with wedge-shaped defects in the right upper lobe consistent with 


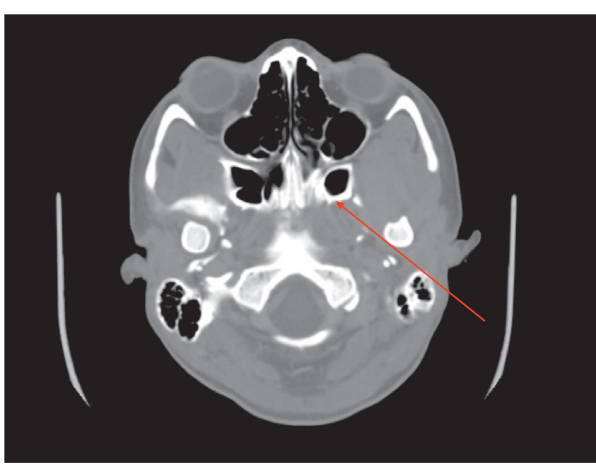

(a)

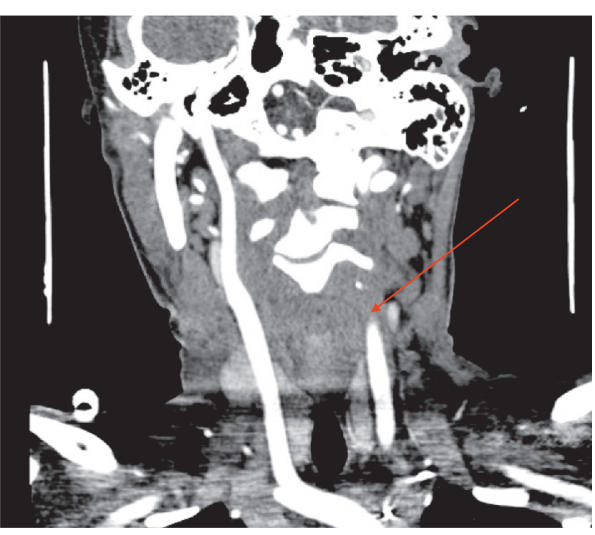

(b)

FIGURE 1: CT angiography. (a) CT angiography venous phase of the head, demonstrating left CST. (b) CT angiography head demonstrating left ICA occlusion.

Medication Regimen

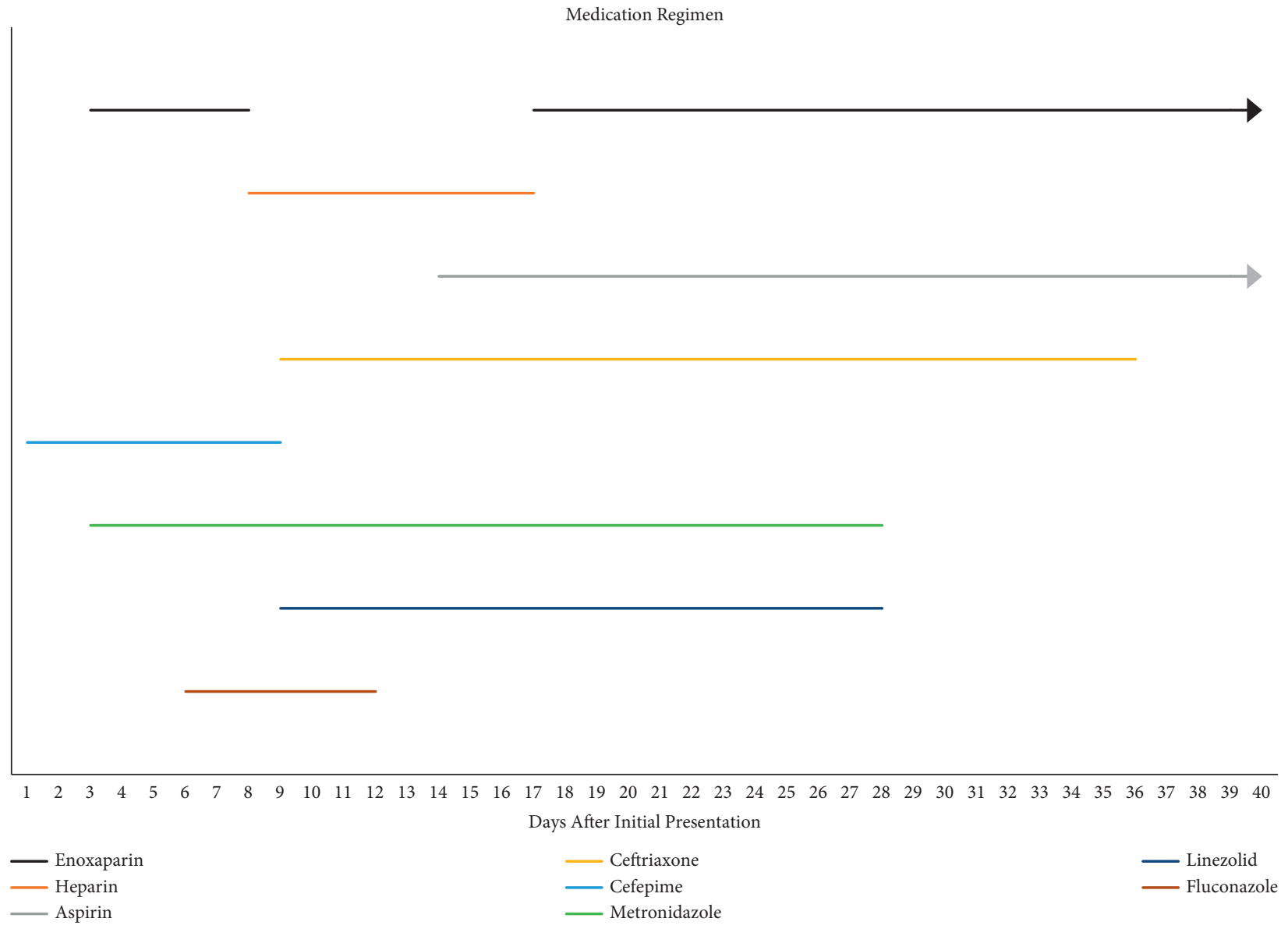

Figure 2: Timeline of the patient's medication regimen.

embolism. Additionally, compute tomography (CT) angiography head, magnetic resonance imaging (MRI) brain, magnetic resonance angiography (MRA) head and neck, and magnetic resonance venography (MRV) head and neck revealed left cavernous sinus thrombosis (CST) with adjacent pachymeningitis, possible cavernous sinus abscess, severe stenosis of the left distal petrous and cavernous internal carotid artery (ICA), focus of severe stenosis in the ICA terminus, and dural thickening and enhancement (Figure 1). The patient underwent two cerebral angiograms; the initial showed left ICA dissecting aneurysm with stenosis with posterior communicating artery collateral flow. The second angiogram four days later showed a complete, left ICA thrombosis.

The patient was managed with a medication regimen including both antibiotics and anticoagulation with age and weight-appropriate dosing. Her medication timeline is summarized in Figure 2. She was managed by various specialties including neurology, neurosurgery, ophthalmology, 
infectious disease, and hematology. The neurosurgery team determined that medical management without surgical intervention was sufficient. She remained on aspirin and enoxaparin after discharge. Unfortunately, her neurological deficits were persistent upon discharge home with outpatient follow-up. Repeat imaging 28 days after initial presentation revealed worsened left ICA occlusion and significant partial resolution of the cavernous sinus thrombosis.

\section{Discussion}

We describe a young female with cavernous sinus thrombosis and multiple cranial nerve palsies resulting from Lemierre's syndrome associated with Streptococcus viridans. Lemierre's syndrome is uncommon, particularly with organisms other than Fusobacteria [10]. A review by Riordan [10] suggests merely $8 \%$ of Lemierre's syndrome cases are due to other pathogens [10]. However, among patients with ophthalmic symptoms, 25\% had Streptococcus infections [5]. Reports of Lemierre's syndrome with various strains of Streptococcus have described complications including CST and isolated cranial nerve palsies, as well as other findings including postseptal cellulitis, pulmonary embolism, pleural effusion, and bibasilar pneumonia [5-9]. While some of these case reports noted isolated trochlear or abducens nerve palsies, they did not report any instances of multiple cranial nerve palsies.

Lemierre's syndrome and its complications can be lifethreatening $[10,11]$. Thus, prompt diagnosis and treatment of the condition are essential. However, there is no clear consensus on treatment for Lemierre's syndrome. Management is typically focused on treating the infection via antibiotics and drainage as necessary [12]. Anticoagulative therapy is controversial. Riordan [10] suggested around $23 \%$ of patients receive anticoagulation [10], while a more current review by Johannesen and Bodtger [13] found up to $64 \%$ are treated with anticoagulation [13]. A systematic review by Adedeji et al. [14] studied 14 patients and found anticoagulation to be a safe therapeutic strategy in Lemierre's syndrome [14]. However, Gore [11] performed a meta-analysis that found anticoagulation did not significantly impact recanalization of thrombosed vessels or mortality rates [11]. Despite this, it has been suggested that decision to anticoagulate may be patient-specific. A review by Kristensen and Prag [15] recommended anticoagulation should only be given in patients at risk of thrombosis extension to the cavernous sinus [15]. Similarly, another review by Moore et al. [16] of 41 patients found $26.8 \%$ of patients improved with addition of anticoagulation and proposed use of anticoagulation in patients with extensive thrombosis [16]. For our patient, anticoagulation was initiated during hospitalization and continued after discharge.

\section{Conclusion}

We describe a pediatric case of Lemierre's syndrome caused by an unusual pathogen, Streptococcus viridans. Our patient developed extensive thrombosis, multiorgan involvement, and unique sequelae of cranial nerves III, IV, and VI palsies. She was treated on a regimen of antibiotics and anticoagulation. This case highlights the importance of a multidisciplinary, team-based approach to patient care. Rapid diagnosis of this rare syndrome is crucial for proper therapeutic management. It is beneficial for neurologists to be aware of Lemierre's syndrome, as there are potentially neurologically devastating sequelae of the syndrome. Moreover, future research and randomized controlled trials are warranted to develop consensus guidelines for anticoagulation and treatment of Lemierre's disease.

\section{Data Availability}

Data for this article were gathered from the patient's medical record.

\section{Conflicts of Interest}

The authors declare that they have no conflicts of interest.

\section{References}

[1] S. P. Dasari and P. Jha, "A systematic review of Lemierre's syndrome with a focus on ophthalmologic complications," Cureus, vol. 12, no. 7, Article ID e9326, 2020.

[2] K. Kuppalli, D. Livorsi, N. J. Talati, and M. Osborn, "Lemierre's syndrome due to fusobacterium necrophorum," The Lancet Infectious Diseases, vol. 12, no. 10, pp. 808-815, 2012.

[3] R. K. Shah, M. M. Wofford, T. G. West, and A. K. Shetty, "Lemierre syndrome associated with group a streptococcal infection," The American Journal of Emergency Medicine, vol. 28, no. 5, pp. e643-e645, 2010.

[4] S. S. Gunatilake, L. G. Yapa, M. Gallala, R. Gamlath, C. Rodrigo, and H. Wimalaratna, "Lemierre's syndrome secondary to community-acquired methicillin-resistant Staphylococcus aureus infection presenting with cardiac tamponade, a rare disease with a life-threatening presentation: a case report," International Journal of Emergency Medicine, vol. 7, no. 39, p. 39, 2014.

[5] Y.-H. Wang, P.-Y. Chen, P.-J. Ting, and F.-L. Huang, "A review of eight cases of cavernous sinus thrombosis secondary to sphenoid sinusitis, including a12-year-old girl at the present department," Infectious Diseases, vol. 49, no. 9, pp. 641-646, 2017.

[6] J. Camacho-Cruz, H. Preciado, N. Beltrán, L. Fierro, and J. Carrillo, "Lemierre's syndrome caused by Streptococcus anginosus presenting as postseptal cellulitis in a pediatric patient," ORL Journal for Otorhinolaryngology and its Related Specialties, vol. 81, no. 4, pp. 234-239, 2019.

[7] M. A. Frizzola and J. H. Hertzog, "Lemierre syndrome in a 22month-old due to Streptococcus pyogenes," Pediatric Emergency Care, vol. 27, no. 11, pp. 1078-1080, 2011.

[8] L. J. Rohowetz, S. M. Gratton, D. Dansdill, C. J. Miller, and S. Dubin, "Cavernous sinus thrombosis caused by Streptococcus constellatus-associated Lemierre syndrome presenting as an isolated abducens nerve palsy," American Journal of Ophthalmology Case Reports, vol. 18, Article ID 100592, 2020.

[9] S. Lee, T. Rutar, F. G. Velez, and A. L. Rosenbaum, "Lemierre's syndrome with fourth nerve palsy," Journal of American Association for Pediatric Ophthalmology and Strabismus, vol. 13, no. 1, pp. 107-108, 2009. 
[10] T. Riordan, "Human infection with fusobacterium necrophorum (Necrobacillosis), with a focus on Lemierre's syndrome," Clinical Microbiology Reviews, vol. 20, no. 4, pp. 622-659, 2007.

[11] M. R. Gore, "Lemierre syndrome: a meta-analysis," International Archives of Otorhinolaryngology, vol. 24, no. 3, pp. e379-e385, 2020.

[12] J. Osowicki, S. Kapur, L. K. Phuong, and S. Dobson, "The long shadow of Lemierre's syndrome," Journal of Infection, vol. 74, pp. S47-S53, 2017.

[13] K. Johannesen and U. Bodtger, "Lemierre's syndrome: current perspectives on diagnosis and management," Infection and Drug Resistance, vol. 9, pp. 221-227, 2016.

[14] A. Adedeji, O. Chukwura, T. Obafemi, S. B. McNulty, and J. P. Reinert, "Anticoagulation strategies in the management of Lemierre syndrome: a systematic review of the literature," The Annals of Pharmacotherapy, vol. 55, no. 5, pp. 658-665, 2021.

[15] L. H. Kristensen and J. Prag, "Human necrobacillosis, with emphasis on lemierre's syndrome," Clinical Infectious Diseases, vol. 31, no. 2, pp. 524-532, 2000.

[16] B. A. Moore, C. Dekle, and J. Werkhaven, "Bilateral Lemierre's syndrome: a case report and literature review," Ear, Nose, \& Throat Journal, vol. 81, no. 4, pp. 234-252, 2002. 